\title{
Proper Management for Morbid latrogenic Retroperitoneal Barium Insufflation
}

\author{
Jalal Vahedian-Ardakani, Shahram Nazerani, Amir Saraee, Ali Sarmast, Ehsan Saraee, \\ Mohammad Reza Keramati \\ Department of Surgery, Iran University of Medical Sciences, Tehran, Iran
}

A barium enema is a diagnostic and therapeutic procedure commonly used for colon and rectum problems. Rectal perforation with extensive intra- and/or extraperitoneal spillage of barium is a devastating complication of a barium enema that leads to a significant increase in patient mortality. Due to the low number of reported cases in recent scientific literature and the lack of experience with the management of these cases, we would like to present our treatment approach to a rare case of retroperitoneal contamination with barium, followed by its intraperitoneal involvement during a diagnostic barium enema. Our experience with long-term management of the patient and the good outcome will be depicted in this paper.

Keywords: Enema; Barium sulfate; Iatrogenic colonic perforation

\section{INTRODUCTION}

A contrast barium enema, or lower gastrointestinal series, is a solution of barium sulfate administered rectally as a diagnostic or therapeutic tool for colorectal diseases. Although the barium enema is a safe study for both benign and malignant conditions of the colon and the rectum, rare serious complications may result. Colonic perforation is one of the most common complications, occurring in approximately $0.02 \%$ to $0.24 \%$ of patients undergoing a barium enema or a double-contrast barium enema $[1,2]$.

Depending on the site of perforation, intra- or extraperitoneal perforation might happen. In cases with intraperitoneal perforation, a combination of barium and fecal matter leads to severe and acute peritonitis and sepsis, followed by intravascular volume depletion. In later stages, if the patient survives the septic shock, dense intraperitoneal adhesions may lead to further serious complica-

Received: May 31, 2014 - Accepted: June 25, 2014

Correspondence to: Mohammad Reza Keramati, M.D.

Department of Surgery, Tehran University of Medical Sciences, Tehran

1951955531, Iran

Tel: +98-912-114-7406, Fax: +98-12-22812468

E-mail:dr_morezak@yahoo.com

(c) 2014 The Korean Society of Coloproctology

This is an open-access article distributed under the terms of the Creative Commons Attribution NonCommercial License (http://creativecommons.org/licenses/by-nc/3.0) which permits unrestricted noncommercial use, distribution, and reproduction in any medium, provided the original work is properly cited. tions $[3,4]$. Extraperitoneal perforations, besides the mentioned sepsis and frank peritonitis, can result in retroperitoneal emphysema and abscess formation $[5,6]$. A mortality of greater than $50 \%$ has been reported for this rare complication, which is called barium peritonitis [2].

Since 1916 when the first case of barium peritonitis was reported by Hullo et al. (quoted from [7]) and later in 1932 when the first similar case during a barium enema was presented, a limited number of case reports have been published in the literature [7]. This is in vivid contrast with the large number of reported iatrogenic colonic perforations due to colonoscopic procedures. Due to the lower incidence of retroperitoneal perorations in these cases, in comparison to the intraperitoneal ones, fewer reports are available in this regard. Therefore, in this paper, we would like to present a very rare case of iatrogenic retroperitoneal barium injection as a result of rectal perforation during a barium enema. $\mathrm{Al}$ though a very limited number of cases with intra- and extraperitoneal rectal perforations following a barium enema have been reported during the past century, our case is the first with a secondary intraperitoneal barium extravasation and peritonitis following a primary retroperitoneal involvement. Despite the high mortality reported for these conditions, we will also depict a proper long-term management with a very good outcome for these patients. This article will review the related literature on this rare condition, as well. 


\section{CASE REPORT}

The Radiology Department requested an emergency surgical consultation for an 84-year-old male patient with sudden lower abdominal discomfort and pain during a diagnostic barium enema. The enema had been scheduled and administered as diagnostic tool for the evaluation of his chronic constipation. The patient had been referred to his gastroenterologist due to a history of obscure chronic constipation for more than 10 years. Although the patient had been consulted for a diagnostic colonoscopy, due to his lack of consent, the procedure had been canceled, and he was then scheduled for a diagnostic barium enema. The enema had been done using a $28 \mathrm{~F}$ plastic rectal tube, followed by rectal infusion of $96 \%$ barium-sulfate solution at a pressure of 100 centimeters of water.

In our first emergent visit, the patient complained of a vague pain in his lower abdomen, which had started immediately following the rectal tube's insertion. He believed that the pain had increased
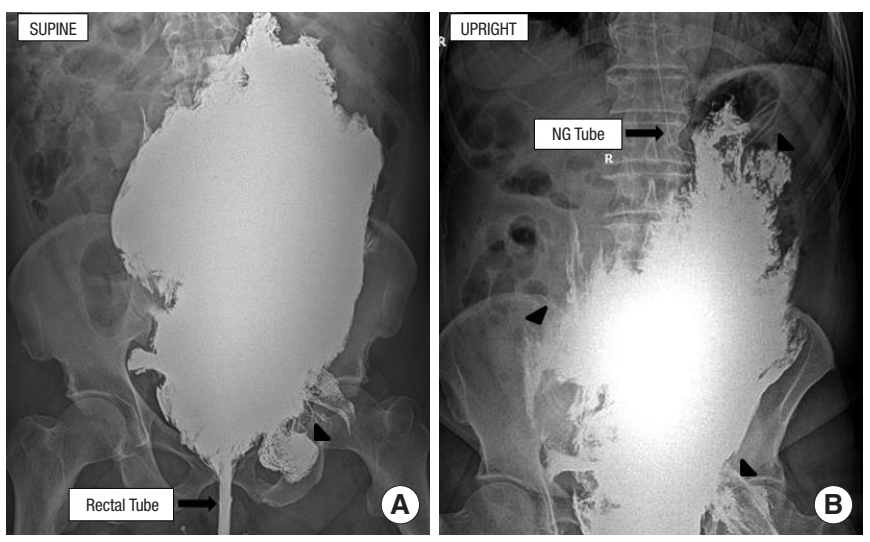

Fig. 1. Abdominal x-rays in supine (A) and upright (B) positions demonstrate the intraperitoneal extravasation of the barium. Arrowheads show extravasation of the barium out of the intestinal tract. during the procedure. Except for a history of chronic heart failure and atrial fibrillation, we found no other previous medical or surgical condition in the patient. He was totally conscious with stable and normal vital signs. Physical examination revealed a generalized abdominal tenderness mostly presented in lower quadrants in addition to insignificant rebound tenderness in the hypogastric region. Digital rectal exam showed a loose, bloody stool. An emergent supine plain abdominal $\mathrm{x}$-ray was taken to evaluate the pattern of the administered barium within the abdomen (Fig. 1). The pattern of contrast extravasation in the $\mathrm{x}$-ray reinforced the possibility of rectal perforation and spillage of the contrast into the surrounding tissues.

After an adequate fluid replacement, resuscitation and prophylactic antibiotics, with the diagnosis of rectal perforation and barium extravasation, the patient underwent an emergent exploratory laparotomy with a midline incision. The laparotomy revealed no site of visible perforation in the distal colon or the sigmoid and upper rectum, but bright drops of barium were detected on the posterior wall of the peritoneum and around the sigmoid mesocolon and the mesoileum (Fig. 2). It seemed that the barium had penetrated from its initial location in retroperitoneum into the intraperitoneal cavity. Despite the adhesive characteristic of barium and its deep penetration into tissues, barium drops were extracted as much as possible, after which a massive irrigation and lavage of the peritoneal cavity was done. A diverting ileostomy was also performed to divert the fecal stream from the distal rectum that was the major site of injury. A tissue sample was also taken from the mesenteric tissue to confirm the possible etiology of peritoneal inflammation. Microscopic evaluation of the sample depicted a fat necrosis and foreign-body reaction within the tissues.

Following the surgery, the patient was transferred to the intensive care unit, and broad-spectrum intravenous antibiotics were administered. Despite the proper hydration and the administration of antibiotics, signs and symptoms of sepsis gradually developed within the next two days. Thereafter, the patient underwent a rigid proctoscopy, which showed a massive inflammation and
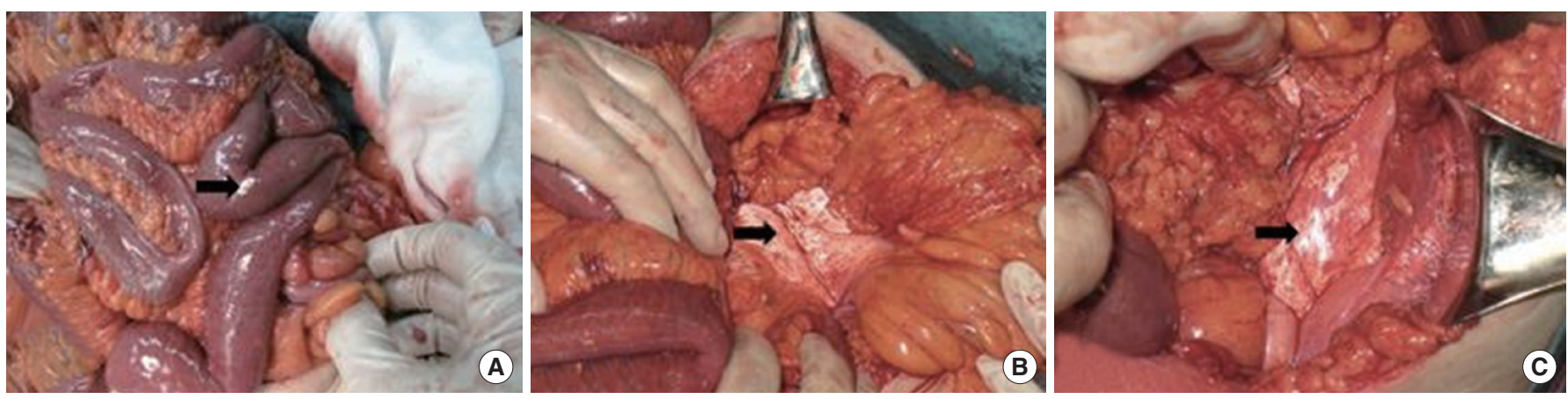

Fig. 2. Intraoperative pictures show barium deposits extravasated into the abdominal cavity from the barium's original location in the retroperitoneal space. (A) Barium can be seen on the small intestinal loops. (B) Barium is also adhesively attached to the small intestinal mesentery. (C) Rectum and intrapelvic viscera have been covered by barium. 


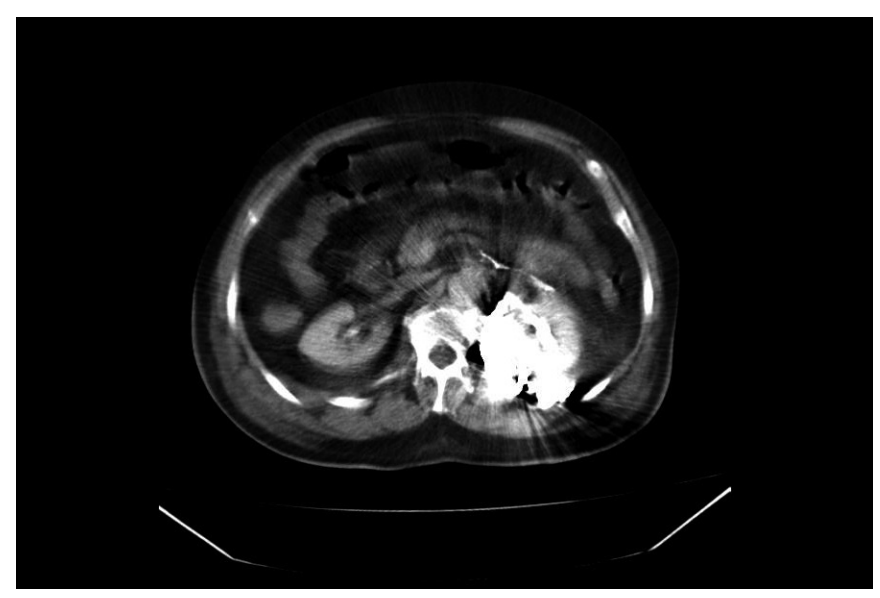

Fig. 3. Abdominopelvic computed tomography scan shows an extensive hyperdense area within the retroperitoneal space on the left side of the pelvis with an extension to the psoas muscles.

blood clots on the posterior wall of the rectum. In order to provide a better drainage system for the presacral space, we also accomplished an open, corrugated, presacral drain.

Although, with close monitoring and proper medication, the general condition of the patient improved and the sepsis was resolved in a period of 10 days following the last procedure, a generalized abdominal pain developed gradually after 14 days. A high fever was also detected. In order to find possible sources of localized infection, such as abscesses within the injured sites, we performed an abdominopelvic computed tomography (CT) scan. The scan demonstrated an extensive hyperdense area within the retroperitoneal space on left side of the pelvis, with an extension to the psoas muscles (Fig. 3). A second laparotomy was performed at that time. During this procedure, a massive dissemination of barium up to the inferior pole of both kidneys was found. The mesentery of the colon and small intestine was covered by barium, and the retroperitoneal tissues were highly fragile and inflamed. In addition to a proper irrigation, a meticulous, but massive, debridement of necrotic tissues within the retroperitoneal area was performed. Two open, corrugated drains were also inserted.

After a week, the patient had stable vital signs. The fever had disappeared, and he had regained his appetite. Both the presacral and the abdominal drains were removed, and the patient began an oral diet gradually. Finally, the patient was discharged after 10 days with a generally good and stable condition. He was also referred to the stoma care center for further support. Thereafter, he was asked to visit with his surgical team on a biweekly basis. Four months later, the diversion ileostomy was closed, and he was advised to do monthly and then bimonthly visits. He had regained his normal life at that time. After two years of postoperative follow-up, he is in very good condition, but his x-rays still show the presence of barium in his retroperitoneal space (Fig. 4).

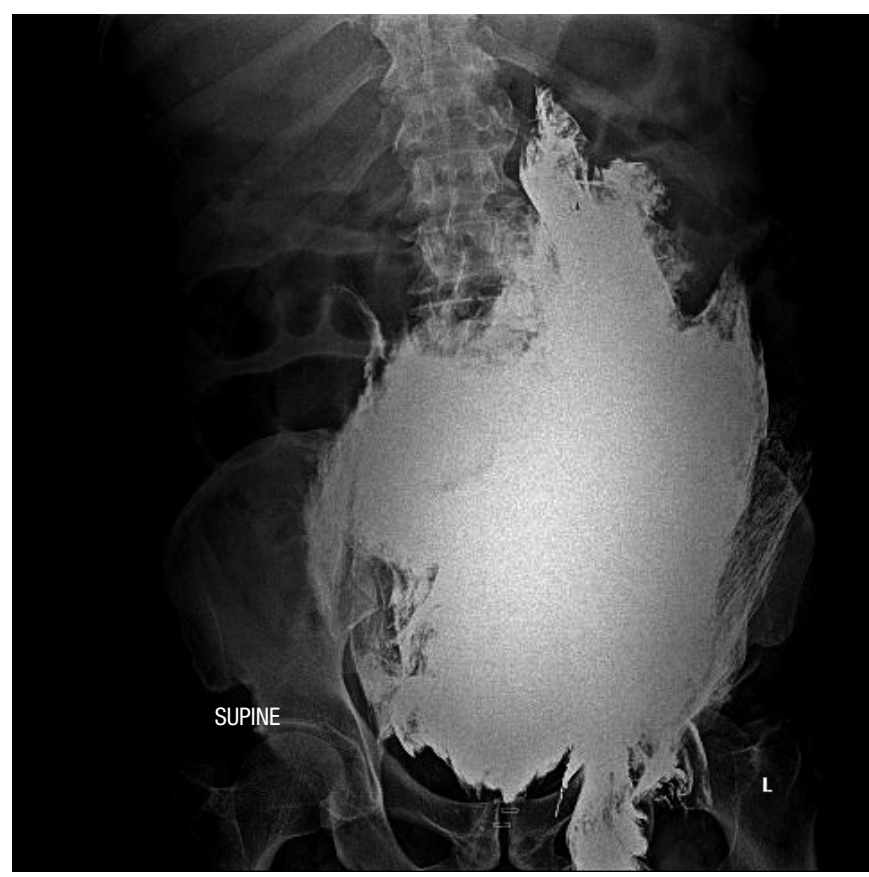

Fig. 4. Abdominal supine $\mathrm{x}$-ray two years after the first admission demonstrates the residual barium. The patient was in good general and health condition at this time point.

\section{DISCUSSION}

\section{Etiology and mechanism}

Although a barium enema is a safe diagnostic study of the colon, perforation of the colon or rectum as a result of injury to the mucosa during this procedure is its most serious complication [8]. Other complications, including barium impaction, water intoxication, allergic reactions and cardiac arrhythmias, have also been reported [1]. Several mechanisms have been suggested for these injuries, including (1) direct trauma from the tip of the catheter or even over inflation of the balloon, (2) excessive transmural pressure after barium insufflation, (3) rectal mucosal damage due to recent instrumentations, colonoscopic procedures or biopsies, and (4) mucosal diseases such as cancer or inflammatory bowel diseases [6]. Our patient did not have any history of recent instrumentation or known colonic disease. Therefore, direct trauma from the enema is the most probable mechanism in our presented case.

\section{Pathophysiology}

For the first time, gross and microscopic responses to intraperitoneal barium injuries in rats were described in detail by Thomas in 1936 [9]. Later, similar histologic findings were reported for extraperitoneal barium injuries in rabbits by Sanders and Kobernick [10]. Based on their findings, the barium starts to agglutinate into fibrin-coated floccules; then, migration of polymorphonuclear cells and hypervascularity of the peritoneum can be observed af- 
ter the first hour of injection. Large barium clumps adhere to the peritoneum, and mononuclear cells appear after three hours. Fibrinous adhesions appear after six hours, which leads to firm adhesions three to five days later. One month later, the barium particles are completely encapsulated by the surrounding tissues, and adhesions are firm and massive. Finally, giant cells surround the encapsulated barium particles after five months. With reference to our case, we found the same gross histological findings during our surgical procedures.

\section{Signs and symptoms}

Depending on the site of injury, different clinical pictures can be expected. Rectal pain is the most common clinical symptom that might appear during introduction of the catheter or after barium or air insufflation. However, this pain can be minimal or even absent. Clinical signs are always minimal, too. Rectal bleeding, emphysema and abdominal tenderness should also raise a suspicion of perforation. A few hours later, tachycardia and fever may develop, which can be followed by a progressive sepsis or septic shock. Peritonitis is a severe, but relatively, late sign [11]. Our patient also presented with a vague and mild lower abdominal pain initially, and signs and symptoms of peritonitis and sepsis developed later.

\section{Classification}

The site and the depth of these injuries are important factors influencing proper diagnosis and treatment strategy. Therefore, various classifications have been described. In general, they can be categorized as intramural (or incomplete) and transmural (or complete) [7]. Peterson et al. [5] have considered anatomic boundaries in their classifications, including (1) infralevator perforations in the anal canal, (2) incomplete perforations, (3) retroperitoneal perforations, (4) transmural perforations into adjacent viscera, and (5) perforations into the free intraperitoneal cavity. Our presented case depicted a transmural retroperitoneal injury with further involvement of the free peritoneal cavity.

\section{Diagnosis}

Plain abdominal $\mathrm{x}$-ray is the method of choice for the initial diagnosis in these patients and has remained the first imaging study. CT has also been proposed to depict the extension of the barium extravasation when the suspicion of perforation arises with high accuracy [12]. Our experience in this case shows that the presence of barium in the intra- or the extraperitoneal space results in an extensive artifact in the images and makes interpretation difficult. In some circumstances, an endoscopy has also been advised for further evaluation of a perforation, especially in cases with an unknown site of perforation or any doubt in the diagnosis [7]. Due to the extensive inflammation within the anal canal and tiny location of the perforation, rigid colonoscopy did not provide any important clue in our case.

For complete intraperitoneal perforations, two pathognomonic radiological findings have been demonstrated: a thin longitudinal layer of barium on both sides of the bowel wall, representing the dissection between the mucosa and the muscularis layer and a transverse striation caused by barium outlining the inner muscle layer. Retroperitoneal perforations have been reported to be mostly localized between the levator muscles and the peritoneal reflection, and due to barium's adherence to the colonic wall, barium extravasation is usually thought to be absent and only air is thought to be able to extravagate further [13]. This is in contrast with the findings in our case in which we found that the barium could go through the retroperitoneal space up to the kidney poles and that it had also penetrated through the peritoneal wall into the intraperitoneal cavity. Barytoma, or barium granulomas, is a term describing the residual barium at the site of the extravasation and along the lymphatic system, which may be visible for many years [14]. Our patient's $\mathrm{x}$-rays after two years clearly show these Barytomas.

\section{Treatment}

Not only is the survival of these patients clearly related to an early diagnosis but also proper management plays an important role. Therefore, in addition to a high degree of suspicion as to the possible diagnosis, the treatment must begin as soon as the diagnosis is made. Adequate fluid replacement and resuscitation, in addition bowel rest and broad-spectrum antibiotics, should be started very soon in these patients. Contradictory opinions regarding an early surgical treatment have been published in the literature. On the one hand, some authors believe that an early laparotomy should be considered for early aggressive evacuation of the barium [1]. On the other hand, others believe that a nonsurgical conservative approach can be considered in cases with intramural or small intraor retroperitoneal perforations with no leakage of barium [7]. In patients with severe peritonitis or gross intraperitoneal perforation immediate surgical intervention is mandatory.

During the surgery, barium should be evacuated and removed as much as possible; then, a thorough lavage and drainage of the abdominal cavity should be done. Debridement of necrotic tissues and drainage of abscesses should also be considered. Usually, primary closure of the perforation site is not recommended, except for fresh infralevator mucosal lesions [5]. A resection of the damaged site and a Hartmann's procedure have been recommended for most of those intraperitoneal lesions [7]. In rare cases with general good condition and minimal spillage of barium, a primary repair or resection and anastomosis with or without a diverting ostomy are recommended. In retroperitoneal perforations, proper drainage and a diverting ostomy are recommended [15]. Our experience also showed that these two steps led to a good long-term outcome in these groups of patients. As a result of massive inflammation around the colon and mesocolon, we preferred an ileostomy over a colostomy for our patient.

\section{Complications}

In addition to sepsis and septic shock, various degrees of early 
abdominal complications may complicate the situation. In patients who survive sepsis, a number of late complications may appear. Ileus is the most common late complication in these patients and has been reported in $30 \%$ of patients; it may be related to adhesive small-bowel obstructions, which are adhesions caused by residual barium in the peritoneal cavity. Other complications, such as retroperitoneal fibrosis, paralytic ileus, ureteral obstruction and hydronephrosis, have been reported after retroperitoneal perforations. Venous intravasation of barium into the portal venous system and mesenteric veins following a barium enema are other rare, but serious, complications [2,7].

In conclusion, despite current advances in proper critical and intensive management of acutely ill patients, iatrogenic barium insufflation into the peritoneal and the retroperitoneal spaces is still a complication with high mortality. In addition to a high degree of suspicion as to a possible diagnosis in cases with early signs and symptoms, rapid diagnosis and management are also the hallmarks of successful treatment. With regard to the high possibility of late complications after discharge, these patients should make regular follow-up visits for a long period of time. Moreover, in each patient, potential complications of this modality have to be weighed against the benefits. Therefore, as a precaution prior to inserting the catheter, a careful digital rectal examination is highly recommended for all patients. In addition, a barium enema should be avoided in patients with known rectal lesions or active colorectal inflammations such as colitis.

\section{CONFLICT OF INTEREST}

No potential conflict of interest relevant to this article was reported.

\section{REFERENCES}

1. Yasar NF, Ihtiyar E. Colonic perforation during barium enema in a patient without known colonic disease: a case report. Cases J 2009; 2:6716.

2. Khan JS, Moran BJ. Iatrogenic perforation at colonic imaging. Colorectal Dis 2011;13:481-93.

3. Nelson RL, Abcarian H, Prasad ML. Iatrogenic perforation of the colon and rectum. Dis Colon Rectum 1982;25:305-8.

4. Cochran DQ, Almond CH, Shucart WA. An experimental study of the effects of barium and intestinal contents on the peritoneal cavity. Am J Roentgenol Radium Ther Nucl Med 1963;89:883-7.

5. Peterson N, Rohrmann CA Jr, Lennard ES. Diagnosis and treatment of retroperitoneal perforation complicating the double-contrast barium-enema examination. Radiology 1982;144:249-52.

6. Tadros S, Watters JM. Retroperitoneal perforation of the rectum during barium enema examination. Can J Surg 1988;31:49-50.

7. de Feiter PW, Soeters PB, Dejong CH. Rectal perforations after barium enema: a review. Dis Colon Rectum 2006;49:261-71.

8. Williams SM, Harned RK. Recognition and prevention of barium enema complications. Curr Probl Diagn Radiol 1991;20:123-51.

9. Thomas JC. The disposal of barium sulphate in the abdominal cavity. J Pathol Bacteriol 1936;43:285-98.

10. Sanders AW, Kobernick SD. Fate of barium sulfate in the retroperitoneum. Am J Surg 1957;93:907-10.

11. de Feiter PW, Soeters PB, Dejong CH. Biphasic development of an intraperitoneal rectum perforation: a rare but serious complication after barium enema. Int J Colorectal Dis 2007;22:719-21.

12. Gardner DJ, Hanson RE. Computed tomography of retroperitoneal perforation after barium enema. Clin Imaging 1990;14:208-10.

13. Gelfand DW, Ott DJ, Ramquist NA. Pneumoperitoneum occurring during double-contrast enema. Gastrointest Radiol 1979;4: 307-8.

14. Vandendris M, Giannakopoulos X. Retroperitoneal barytoma. Urology 1981;17:358-9.

15. Levy MD, Hanna EA. Extraperitoneal perirectal extravasation of barium during a barium enema examination: natural course and treatment. Am Surg 1980;46:382-5. 